\title{
Morbidade de Membros Superiores e Qualidade de Vida após a Biópsia de Linfonodo Sentinela para o Tratamento do Câncer de Mama
}

\author{
Upper Limb Morbidity and Quality of Life After Lymph Node Sentinel Biopsy \\ for Breast Cancer Treatment
}

Fernanda Saltiel Barbosa Velloso', Alexandre de Almeida Barra², Rosângela Corrêa Dias ${ }^{3}$

\section{Resumo}

O câncer de mama é o segundo tipo de câncer mais frequente no mundo e o mais comum entre as mulheres. A tendência atual da abordagem terapêutica é oferecer a mínima intervenção que garanta o controle da doença, menos morbidades e preserve a qualidade de vida (QV). A técnica de biópsia de linfonodo sentinela (BLS) se baseia nessa premissa. Este artigo teve por objetivo revisar a literatura quanto à prevalência de morbidades relativas aos membros superiores e à QV entre pacientes submetidas à BLS. Foram consultadas as bases de dados Pubmed, Cochrane, Scielo e Lilacs entre os anos de 1997 e 2008. Os descritores de assunto utilizados foram Breast neoplasms, Sentinel lymph node biopsy, Morbidity e Quality of life. Foram encontrados 76 artigos e selecionados 22. Os resultados mostraram que as morbidades mais frequentemente estudadas foram alterações de sensibilidade (prevalência entre $7 \%$ a $88 \%)$, dor (3,5\% a 39\%), linfedema ( $0 \%$ a $22 \%)$, redução da amplitude de movimento do ombro (3\% a 16\%), limitação na realização das atividades de vida diária (AVDs) $(7,8 \%$ a 27,8\%) e interferência na QV. Após 36 meses, as morbidades de membro superiores pouco interferiram na QV. As pacientes se preocuparam mais com o medo da recidiva. O tempo transcorrido da cirurgia e os instrumentos utilizados para a coleta dos dados explicam a variabilidade na prevalência de sintomas. Faltam estudos de acompanhamento de longo prazo para avaliar a evolução das morbidades e que avaliem a QV de pacientes brasileiras.

Palavras-chave: Neoplasias da mama; Biópsia de linfonodo sentinela; Morbidade; Qualidade de vida

${ }^{1}$ Mestre em Ciências da Saúde pelo Instituto de Previdência Social de Minas Gerais (IPSEMG), Professora assistente do curso de Fisioterapia do Centro Universitário de Belo Horizonte (MG)

${ }^{2}$ Doutor em Medicina pela UFMG. Médico Mastologista do IPSEMG. Professor do Mestrado de Ciências de Saúde do IPSEMG

${ }^{3}$ Doutora em Ciências da Reabilitação pela Universidade Federal de São Paulo. Professora Associada do departamento de Fisioterapia da Escola de Educação Física, Fisioterapia e Terapia Ocupacional da UFMG

Endereço para correspondência: Av. Engenheiro Carlos Goulart, 1.495 - Buritis - Belo Horizonte (MG), Brasil - CEP: $30455-700$.

E-mail: fernandasaltiel@gmail.com 


\section{INTRODUÇÃO}

O câncer de mama é o segundo tipo de câncer mais frequente no mundo e o mais comum entre as mulheres. A cada ano, cerca de $22 \%$ dos casos novos de câncer em mulheres são de mama ${ }^{1}$.

As características do procedimento cirúrgico e terapia adjuvante podem trazer complicações funcionais para a articulação do ombro ipsilateral à mama que recebeu o tratamento. Quanto mais extensa for a cirurgia, maiores serão as chances de morbidade ${ }^{2,3,4}$. A abordagem cirúrgica tem se modificado radicalmente nos últimos dez anos. Entre os avanços, está a técnica de Biópsia do Linfonodo Sentinela (BLS), desenvolvida na década de 1990, cujo primeiro estudo aleatorizado data de $2003^{5,6}$. A tendência atual da abordagem terapêutica é oferecer a mínima intervenção que garanta o controle da doença, tomando o cuidado de sempre limitar as morbidades e preservar a qualidade de vida $(Q V)^{6}$. A opção pela BLS se baseia na redução da morbidade, sem, no entanto, representar maior mortalidade se comparada ao esvaziamento axilar ${ }^{7}$. No entanto, os aspectos negativos da BLS são subestimados, enquanto as complicaçōes da linfadenectomia axilar total (LAT) são superestimadas, visto o entusiasmo científico que acompanha o desenvolvimento da nova técnica ${ }^{8}$.

$O$ objetivo desta revisão foi identificar a prevalência das morbidades funcionais e a $\mathrm{QV}$, decorrentes da cirurgia conservadora da axila (BLS) para o tratamento do câncer de mama em mulheres em médio-longo prazo (após seis meses da cirurgia).

\section{MATERIAL E MÉTODOS}

A revisão de artigos foi realizada nas línguas inglesa e portuguesa nas bases de dados Pubmed, Cochrane, Scielo e Lilacs, utilizando os seguintes descritores de assunto: Breast neoplasms, sentinel lymph node biopsy, morbidity e quality of life, combinados entre si no período de 1997 a 2008. A opção por esses bancos de dados se justifica por serem conhecidos e muito utilizados por acadêmicos, docentes e profissionais da área de saúde e por indexarem periódicos de boa qualidade. Foram selecionados para esta revisão artigos que trataram da morbidade de membros superiores e qualidade de vida em mulheres submetidas à técnica BLS para o tratamento do câncer de mama. Foram excluídos artigos que tratassem da descrição da técnica cirúrgica e anatomopatológica, análise de eficácia, índice de recidiva, sobrevida, sistema de estadiamento do câncer de mama baseado na BLS, mapeamento anatômico da drenagem linfática da mama, câncer de mama em homem, artigos de revisão não sistemática e comentários. A base de dados Cochrane foi pesquisada para identificar revisões sistemáticas sobre o tema. Foi encontrado apenas um estudo clínico aleatorizado na Cochrane, que fora também identificado no Pubmed. No Pubmed e Cochrane, foram encontrados 72 artigos, sendo selecionados 21; no Lilacs, foi encontrado um artigo que foi selecionado. Foram encontrados três artigos na base de dados Scielo, mas nenhum abordava as morbidades de pacientes pós-BLS separadamente da abordagem axilar (Figura 1). Portanto, foram selecionados, no total, 22 artigos.

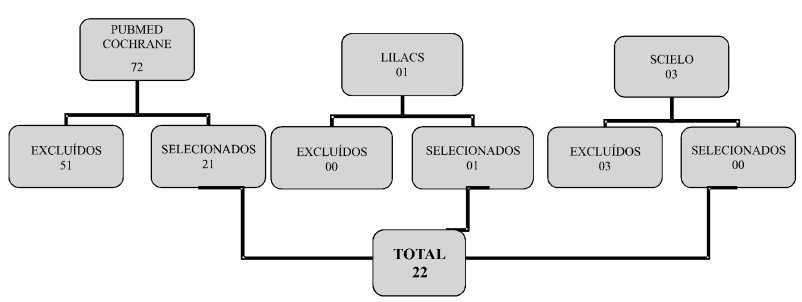

Figura 1. Diagrama dos artigos encontrados nas bases de dados consultadas

Todos os trabalhos identificados na literatura apresentaram análise comparativa entre as morbidades após a LAT e aquelas observadas entre pacientes submetidas à BLS. Tendo em vista o objetivo deste artigo, serão apresentados os dados relativos às pacientes submetidas à LAT apenas quando for necessário para explicar a variação na morbidade entre os indivíduos que realizaram a BLS.

\section{MORBIDADES RELACIONADAS AO SISTEMA MUSCULOESQUELÉTICO ASSOCIADAS AO TRATAMENTO DO CÂNCER DE MAMA}

As morbidades relacionadas ao sistema musculoesquelético, relatadas na literatura como estando associadas ao tratamento do câncer de mama, são a parestesia da região axilar e da parede lateral do tórax, dor, aumento do volume do membro superior, a redução da amplitude de movimento do ombro, limitação na realização das atividades de vida diária (AVDs) e a interferência na $\mathrm{QV}^{2,3,4,8,9,10,11}$. Como a técnica BLS tem sido recentemente adotada para o tratamento cirúrgico do câncer de mama, a maioria dos estudos apresenta resultados relativos ao acompanhamento de curto-médio prazo (até 36 meses) 2,3,4,8,9,10,11,12,13,14,15,16,17,18,19,20,21,22,23,24,25. Apenas um estudo acompanhou as mulheres por um período de cinco anos ${ }^{26}$. A seguir, serão apresentadas as principais evidências científicas relacionadas a cada um dos desfechos de interesse desta revisão. 


\section{ALTERAÇÃO DA SENSIBILIDADE}

A morbidade relatada como mais frequente entre pacientes submetidas à BLS é a alteração da sensibilidade da região da axila e mama. Na cirurgia, é provável que aconteça a transecção dos nervos intercostobraquial ou intercostal cutâneo inferior, porque a parte inferior da axila, localização anatômica desses nervos, é a região em que mais frequentemente se localiza o linfonodo sentinela ${ }^{24}$. A sua transecção é traduzida em alteração da sensibilidade da região axilar e interna do braço. A prevalência de sintomas varia entre $7 \%$ e $88 \%$ de acordo o tempo transcorrido da cirurgia e com a característica da sensação avaliada.

Ronka et al. ${ }^{16}$ encontraram relatos de sensações estranhas no braço em 19\%, na mama em $44 \%$ e dormência leve no braço em $7 \%$ das 43 mulheres submetidas à BLS um ano após a cirurgia. Foi utilizado filamento de von Frey para a coleta de dados. Os autores afirmam que os cirurgiōes tomaram precaução para preservar o nervo intercostobraquial, mas não informam quantas pacientes efetivamente não tiveram a transecção. Burak et al. ${ }^{24}$, utilizando um questionário por eles desenvolvido, encontraram prevalência de 16,7\% de parestesia após, em média, $13 \pm 4,5$ meses da cirurgia. Não houve preocupação dos cirurgiōes em preservar os nervos sensitivos da axila. Dentre as 86 pacientes estudadas após um ano da BLS por Purushotham et al. ${ }^{19}$, $23 \%$ delas relataram algum tipo de parestesia. Os autores não apresentaram de forma detalhada os dados das pacientes submetidas à BLS com linfonodo negativo. A sensibilidade foi avaliada através de autorrelato e da medida objetiva de sensibilidade dolorosa e tátil. Foi relatado quando houve a preservação do nervo intercostobraquial apenas no grupo submetido à LAT.

Temple et al. ${ }^{23}$ também relatam que a maioria das pacientes submetidas à BLS apresentou sintomas sensoriais. Os dois sintomas mais prevalentes no pósoperatório imediato foram o aumento de sensibilidade presente em $88 \%$ das mulheres avaliadas, e o dolorimento presente em $80 \%$ delas. Após 12 meses, 52\% ainda relatavam aumento da sensibilidade e $48 \%$ dolorimento. Não foi relatada a localização dos sintomas. Os dados foram coletados utilizando-se o questionário Breast Symptom Assessment Scale (BSAS). A melhora mais intensa foi vista nos primeiros três meses. Mulheres mais jovens, independente do tipo de cirurgia, apresentaram mais sintomas sensoriais. Provavelmente porque sejam mais sensíveis ao desconforto causado pela cirurgia axilar. Outras explicações são as diferenças relacionadas à idade quanto às expectativas e experiências prévias de vida.

Ao acompanharem pacientes antes e seis semanas, 12 e 24 meses após a cirurgia de BLS e as compararem com aquelas que realizaram LAT, Rietman et al. ${ }^{9,14,15}$ publicaram três estudos analisando aqueles três períodos de acompanhamento com as medidas pré-cirúrgicas. Foi encontrada menor prevalência de sintomas sensitivos após 12 meses. A dormência às seis semanas acometeu $64 \%$ das pacientes, aos 12 meses, $17 \%$ e aos 24 meses, $18 \%$. Isso indica que os sintomas após um ano, quanto à sensibilidade, parecem pouco se alterar. A presença de dormência foi relata pela paciente durante o exame clínico.

Após 20 meses de cirurgia (10 a 31 meses), Barranger et al. ${ }^{17}$ conduziram um estudo transversal e encontraram relatos de parestesias, disestesias e dor em 5,9\%, 5,7\% e $21,2 \%$, respectivamente, nas 54 mulheres submetidas à cirurgia conservadora de mama associada à BLS. Os dados foram coletados via telefone, utilizando um questionário desenvolvido a partir dos questionários European Organisation Research and Treatment of Cancer Quality of Life Questionnaire (EORTC-QLQC30) e European Organisation Research and Treatment of Cancer Quality of Life Questionnaire - Breast Module (EORTC-BR23).

O questionário BSAS foi utilizado no estudo de Baron et al. ${ }^{26}$ para avaliar a sensibilidade, e ficou demonstrado que a sensação mais prevalente após cinco anos, tanto no grupo de pacientes que realizaram BLS (33\%) quanto no grupo que fez a LAT (40\%), foi a percepção de maior sensibilidade. Outras sensaçōes comuns no grupo BLS foram sensações de pontadas (34\%), dolorimento (27\%), dor $(27 \%)$ e repuxões $(27 \%)$. Embora as alterações de sensibilidade tenham persistido por cinco anos, a maior redução das queixas aconteceu no período de três meses da cirurgia. Entre as pacientes submetidas à mastectomia, 40\% ainda tinham sensação fantasma da mama após cinco anos. Mas o nível de gravidade e sofrimento causado por ela foi considerado baixo.

A variedade de instrumentos utilizados para se avaliar a sensibilidade e a diferença no tempo de acompanhamento dos estudos dificulta a comparação dos resultados. Ainda assim, todos os estudos indicam que a alteração na sensibilidade perdura mesmo após cinco anos da cirurgia. A maior recuperação da sensibilidade acontece no primeiro ano.

\section{DOR}

A dor é um construto multidimensional e pode ser descrita quanto à intensidade, qualidade e localização no $\operatorname{corpo}^{27}$. A ocorrência de dor após a BLS variou entre $3,5 \%$ e $39 \%$ a depender do instrumento utilizado, da caracterização da deficiência sensorial, do tempo de acompanhamento, do tipo de abordagem cirúrgica da mama e da terapia adjuvante adotada. De qualquer forma, a intensidade da dor experimentada pelas pacientes, de modo geral, é baixa. 
A dor ao movimento esteve presente em 9,7\%, 3,3\%, $6,4 \%, 3,4 \%$ e $3,1 \%$ das pacientes submetidas à BLS após um, três, seis, 12 e 18 meses da cirurgia, respectivamente, no estudo de Fleissig et al. ${ }^{8}$. Magaldi et $a l^{4}{ }^{4}$, usando questionário com respostas dicotômicas ( $\operatorname{sim} /$ não) elaborado para a pesquisa, encontraram $10 \%$ de relato de dor pelas pacientes entre seis e 18 meses após a cirurgia. Em ambos os estudos, a intensidade da dor não foi quantificada.

A intensidade da dor sentida pelas pacientes pós BLS é baixa. Um estudo longitudinal prospectivo não aleatorizado realizado por Peitinger et al. ${ }^{25}$ quantificou a dor em mulheres uma semana após a alta hospitalar e nove a 12 meses após a BLS, utilizando a escala visual analógica (EVA), que gradua a intensidade da dor numa escala de 0 a $10 \mathrm{~cm}$. Quanto maior a intensidade da dor, maior o valor obtido na escala. Foram encontrados valores de $0,68 \pm 1,45$ e $0,68 \pm 1,13$, respectivamente. Todas as pacientes realizaram cirurgia conservadora da mama. Arnaud et al..$^{20}$ também utilizaram a EVA para avaliar a dor após um ano de cirurgia de BLS versus LAT. A média dos escores entre todas as 113 pacientes que realizaram BLS foi de $1,03 \pm 2,09$. Vinte mulheres (23\%) haviam realizado mastectomia. As mulheres com escolaridade mais baixa (abaixo do $3^{\circ}$ grau), que moravam com parceiro, que realizaram mastectomia, que fizeram LAT imediata, que apresentaram complicaçôes póscirúrgicas e que realizaram quimioterapia (QT) apresentaram maior escore na EVA. Também, as pacientes mais jovens tiveram escores mais elevados de dor.

Nos estudos de Rietman et al..$^{9,14,15}$, a dor aumentou em $1,1 \pm 2,1 \mathrm{~cm}$ medida pela EVA após seis semanas da cirurgia, aos 12 meses já havia se reduzido $(0,2 \mathrm{~cm} \pm 1,2)$ e aos 24 meses a diferença em relação à medida précirúrgica foi de $0,6 \pm 18,4$. Partindo do pressuposto que não havia dor antes da operação (os autores não comentam a esse respeito), a intensidade da dor foi baixa. Em seus estudos, 26\% das pacientes realizaram mastectomia. Embora a comparação entre os tipos de cirurgia da mama não tenha sido objeto do estudo, a mastectomia parece ter interferido apenas na escala Imagem Corporal do questionário de QV utilizado (EORTC-BR23).

Ronka et al. ${ }^{16}$ encontraram $28 \%$ das 43 mulheres avaliadas após um ano da BLS com relato de dor no braço, sendo que $16 \%$ a classificaram como leve e $12 \%$ como de intensidade moderada. A dor na mama esteve presente em 37\% da amostra, sendo considerada leve por $30 \%$. Nesse estudo, $19 \%$ das participantes foram submetidas à mastectomia. Os distúrbios sensoriais foram caracterizados em dor, dormência e sensaçōes estranhas no braço ou na mama.
Um estudo transversal com 92 pacientes submetidas à BLS após três anos da cirurgia (33 a 41 meses), conduzido por Leidenius et al. ${ }^{18}$, detectou que $39 \%$ das mulheres queixaram-se de dor na região da mama, sendo considerada moderada e grave por apenas $5 \%$ delas. Onze pacientes $(12 \%)$ relataram dor no membro superior, e todas a quantificaram como leve. Os dados foram coletados através de questionário desenvolvido pelos próprios autores, o qual continha três níveis para categorizar a intensidade da dor/alteração sensorial (leve, moderada ou grave). Não houve diferença quanto às morbidades, comparando-se pacientes submetidas à mastectomia $(9 \%)$ com os que realizaram cirurgia conservadora de mama. Os autores dividiram as sensaçōes em apenas duas categorias: dor ou alteraçōes sensoriais.

\section{LINFEDEMA}

Embora existam vários critérios aceitos para se diagnosticá-lo, não há concordância sobre o que se constitui de fato em linfedema, dificultando a comparação entre os estudos ${ }^{2,12,13}$. Thomas-Maclean et al. ${ }^{12}$, medindo a circunferência dos membros superiores de uma amostra de mulheres, após o tratamento cirúrgico para câncer de mama (BLS e LAT), encontraram valores entre $9 \%$ e $16 \%$ a depender do critério utilizado para definir sua presença. Assim, a prevalência de linfedema pós-BLS tem sido relatada variando de $0 \%$ a $22 \%$. Também não há período definido acima ou abaixo do qual tenha sido observada maior ocorrência de linfedema ${ }^{2,4,8,10,13}$.

Alguns fatores de risco estão associados ao aumento dessa taxa, como o número de nódulos linfáticos dissecados (acima de cinco), radioterapia (RT) axilar, índice de massa corporal (IMC) acima de $30^{12}$ e tempo transcorrido da cirurgia, pois o linfedema tende a acontecer mais tardiamente ${ }^{10,11}$. Schulze et al..$^{13}$, em um estudo retrospectivo, calcularam a volumetria dos membros superiores através da medida da perimetria, usando a fórmula do cone truncado. De suas 19 pacientes, que puderam ser avaliadas em longo prazo, com média de idade de 63 anos e IMC de 26,1, submetidas à BLS, 15,8\% apresentaram linfedema com diferença entre $10 \%$ e $20 \%$ entre membros após, em média, 31,4 meses da cirurgia. Nenhuma apresentou valores superiores a $20 \%$. Quando excluídas as pacientes submetidas à RT axilar, o índice de linfedema foi nulo. Armer et al..$^{10}$ encontraram prevalência de $22,2 \%$ de linfedema, em média 9₫3,9 meses, após a BLS em mulheres tratadas de câncer de mama em um centro de tratamento no meio-oeste norte-americano. Os autores usaram a perimetria acima de $2 \mathrm{~cm}$ para caracterizar a presença de linfedema. A taxa elevada pode ter sido 
associada ao fato da amostra ter incluído mulheres operadas durante o período de treinamento dos cirurgiōes na técnica BLS. Ao longo de 12 meses de acompanhamento, houve aumento médio de volume do membro superior do lado da BLS em relação ao contralateral em $20,4 \mathrm{ml}$, com valores maiores entre o terceiro e sexto mês no estudo de Purushotham et al. ${ }^{19}$. Considerando o ponto de corte de $150 \mathrm{ml}$ apresentado por Thomas-Maclean et al. ${ }^{12}$ para caracterizar a presença de linfedema, os dados apontam para ausência de aumento do volume do membro que represente importância clínica. Achados semelhantes foram encontrados por Rietman et al. ${ }^{9,14,15}$. Houve aumento da circunferência do braço e antebraço em cerca de $1 \mathrm{~cm}$ às seis semanas, que se reduziu aos 12 meses. Aos 24 meses, os autores relatam redução de $2 \mathrm{ml}$ no volume do braço em relação às medidas pré-cirúrgicas. Temple et al..$^{23}$ também não encontraram linfedema entre as 171 pacientes submetidas à BLS após um ano através da circunferência medida $10 \mathrm{~cm}$ acima e abaixo do olecrano. Os autores alertam para o fato de o linfedema ocorrer mais tardiamente.

Magaldi et al. ${ }^{4}$ relataram que apenas uma paciente (2\%) desenvolveu linfedema após 18 meses da cirurgia. $\mathrm{O}$ método de medida utilizado foi a comparação entre os membros superiores da perimetria em oito pontos, sendo considerado ponto de corte de $2 \mathrm{~cm}$. Em seu estudo, $32 \%$ das mulheres foram submetidas à RT axilar e os autores não relatam se este foi o caso da paciente acometida. Langer et al. ${ }^{2}$ encontraram 3,5\% de ocorrências de linfedema medidas por perimetria em apenas dois pontos dos membros superiores após 31 meses da cirurgia, também utilizando o ponto de corte de $2 \mathrm{~cm}$. As pacientes realizaram RT apenas na mama. Esses autores afirmam que a combinação das medidas do membro superior com a apreciação de sintomas representa a avaliação clínica mais confiável para se identificar a presença do linfedema após o câncer de mama. Fleissig et al. ${ }^{8}$ encontraram relato de sensação de edema em 2,4 e 3,5\% das pacientes após 12 e 18 meses, respectivamente. Em sua pesquisa, apenas 8,8\% dos pacientes realizaram RT axilar. Leidenius et al. ${ }^{18}$ não encontraram diferenças superiores a $2 \mathrm{~cm}$ após três anos entre o membro superior ipsilateral à cirurgia de BLS e o contralateral, mas encontraram sinais clínicos de linfedema em $1 \%$ das pacientes, e $5 \%$ delas relataram sensação de edema no braço, sendo que $1 \%$ o classificou como grave. Seus resultados são corroborados por Ronka et al. ${ }^{16}$ quanto à diferença entre medida objetiva $\mathrm{e}$ subjetiva do linfedema. Estes autores encontraram 2\% de mulheres após um ano de acompanhamento que desenvolveram linfedema com diferença entre membros em torno de $10 \%$ e $20 \%$, porém, ao avaliar o autorrelato sobre a sensação de linfedema, $23 \%$ relataram sentir aumento do volume da região da mama e $12,5 \%$ na regiāo do braço. Ambos os grupos de autores discutem os achados considerando que o autorrelato pode superestimar os dados, quando usando questionários, porque os distúrbios sensoriais, como dormência e formigamento, decorrentes da lesão do nervo intercostobraquial, podem ser confundidos com a sensação de linfedema. Por outro lado, Armer et al. ${ }^{10}$ afirmam que o líquido intersticial aumenta entre $50 \%$ e $100 \%$ antes que o edema seja percebido clinicamente. Portanto, a sensação de aumento da pressão intersticial precede um aumento mensurável em volume. Ainda segundo os autores, alguns profissionais de saúde defendem que o relato subjetivo de linfedema deve garantir a intervenção terapêutica precoce. Outros sugerem que as sensaçóes de peso, dor e dificuldade no movimento do braço deveriam ser um índice para se avaliar a eficácia do tratamento do linfedema. Eles também afirmam que o linfedema pode acontecer até 30 anos após o tratamento para o câncer de mama.

\section{AMPLITUDE DE MOVIMENTO DO OMBRO (ADM)}

A BLS pouco interfere na movimentação do ombro. A RT parece ser o fator que mais contribuiu para a restrição da amplitude de movimento do ombro (ADM), embora apenas um estudo tenha analisado essa associação. A RT axilar quase não é indicada atualmente, e a RT na mama, mesmo que em menor grau, pode provocar morbidades no braço como linfedema e alterações da $\mathrm{ADM}^{12}$.

Os resultados dos estudos de Rietman et al.,14,15 mostram que houve limitação dos movimentos de flexão, abdução e rotação externa do ombro nos três momentos analisados (seis semanas, 12 e 24 meses após a cirurgia, comparando-se com dados pré-operatórios), mas as diferenças foram significativas entre os grupos apenas após um ano, com o grupo submetido à BLS apresentando menos restrições na amplitude de movimento do ombro aos 12 e 24 meses. No entanto, os autores não apresentaram análise estatística comparando as características funcionais antes e após a cirurgia, analisando apenas o grupo submetido à BLS. Essas informaçōes foram fornecidas somente no acompanhamento após 24 meses, indicando que a limitação na abdução combinada com a rotação externa no grupo BLS foi de 3,5 $\pm 8,0$ com significância estatística em relação ao período pré-operatório. A RT foi iniciada após a sexta semana. A RT axilar foi considerada fator preditivo de redução da ADM e ocorrência de linfedema nas análises multivariadas, porém não foram apresentadas quantas pacientes haviam-na realizado no grupo BLS. 
Da mesma forma, no estudo de Purushotham et al. ${ }^{19}$, foi encontrada pouca redução da ADM no grupo de mulheres acompanhas no pré e em quatro momentos (um, três, seis e 12 meses) após a cirurgia de BLS ao longo de um ano. No primeiro mês, houve diferença significativa no arco de movimento de abdução do ombro e de flexão no terceiro mês ao comparar o grupo BLS com o LAT. No décimo segundo mês, o movimento apresentando maior limitação foi o de flexão do ombro, em que houve perda de 6,70. Não foi informado se essa diferença foi estatisticamente significativa. Também não apresentaram o ponto de corte considerado para determinar a presença de limitação. Setenta e nove pacientes $(92 \%)$ foram submetidas à RT, mas não houve informaçōes sobre a irradiação da axila. Os dados foram analisados, considerando a intenção de tratar.

Ronka et al. ${ }^{16}$ encontraram limitação de ADM de flexão, abdução e rotação lateral acima de $10^{\circ}$ após um ano da cirurgia em apenas $10 \%, 7 \%$ e $16 \%$ das pacientes de BLS, respectivamente. Houve diferença significativa entre o grupo submetido à BLS e LAT quanto à limitação dos movimentos de flexão e abdução apenas duas semanas após o procedimento cirúrgico. Os autores ainda acompanharam as pacientes após três, seis e 12 meses da cirurgia. Entre as que foram submetidas à BLS, 2\% realizaram RT axilar.

Em estudo brasileiro com mulheres com média de idade de 61,1 anos, Magaldi et al. ${ }^{4}$ encontraram ADM de $100^{\circ}$ de abdução do ombro no primeiro dia pósoperatório, e aos três, seis e 18 meses após a cirurgia a medida aproximou-se da amplitude máxima de $180^{\circ}$ para o movimento estudado $\left(167^{\circ}, 171^{\circ}\right.$ e $168^{\circ}$, respectivamente). Não houve diferença estatística entre as medidas após o terceiro mês de acompanhamento, indicando que a recuperação maior ocorreu neste período. Trinta e dois por cento das pacientes receberam RT, mas não foi informado se a axila foi irradiada. Os autores não mediram outros movimentos dos ombros.

Após 31 meses da BLS, Schulze et al..$^{13}$ não encontraram restrição de abdução acima de $10^{\circ}$ entre as pacientes, mas encontraram apenas 3\% com restrições na adução. Houve irradiação da mama em $61,3 \%$ da amostra. Passados 36 meses da cirurgia, Leidenius et al. ${ }^{18}$ encontraram $16 \%$ das pacientes com restrição de ADM acima de $10^{\circ}$ no movimento de flexão, mas não nos movimentos de abdução, rotação medial e lateral. No grupo BLS, 91\% fizeram RT na mama ou tórax. Nenhuma recebeu RT axilar.

Langer et al. ${ }^{2}$ encontraram 3,5\% da sua amostra com restrição de ADM quantificada pela goniometria, após 31 meses (mediana) da intervenção. Não há menção de quais movimentos apresentaram limitação. Todas as pacientes receberam RT apenas na mama.

Deve-se ressaltar que a goniometria não é capaz de detectar mudanças inferiores a $5^{\circ}$, mesmo quando feita pelo mesmo examinador, além de apresentar baixa reprodutibilidade interexaminador, indicando a necessidade de se realizar teste de confiabilidade das medidas ${ }^{28}$.

O movimento do ombro mais relatado como restrito após um ano da cirurgia foi a flexão. Ainda assim, poucas foram as pacientes que continuaram apresentando restrição de movimento em médio prazo. A limitação é maior no período imediatamente após a operação, e a maior recuperação ocorre nos primeiros três meses. Quanto aos demais movimentos, os dados são conflitantes.

\section{LIMITAÇÃO DE ATIVIDADES DE VIDA DIÁRIA (AVDs)}

Menos de $50 \%$ dos pacientes relatam limitação na realização das atividades cotidianas como resultado das deficiências sensoriais, dos sistemas musculoesquelético e linfático que podem ocorrer após a BLS. O retorno às atividade se dá no primeiro ano da cirurgia.

Ronka et al. ${ }^{16}$ relatam que $17 \%$ das mulheres, após um ano, informaram que as morbidades interferiram na realização das AVDs, $19 \%$ nas atividades de lazer e $21 \%$ no trabalho, embora de forma leve. Três pacientes (7\%) queixaram dificuldades em dormir do lado operado. Apenas 7,8\% das 180 mulheres avaliadas no estudo de Schijven et al. ${ }^{22}$ apresentaram dificuldade na realização das tarefas diárias. A maioria das pacientes $(86,9 \%)$ foi acompanhada entre um e dois anos da cirurgia. No entanto, $21,4 \%$ das pacientes estavam ou já haviam realizado fisioterapia, o que pode ter reduzido os sintomas e favorecido à realização das atividades cotidianas. Mansel et al. ${ }^{3}$, no primeiro grande estudo multicêntrico aleatorizado e controlado comparando LAT com BLS, mostraram que, em três meses, 416 pacientes (94\%) submetidas à BLS retornaram às suas AVDs e, após 12 meses, 413 pacientes (79\%) às suas atividades econômicas. Swenson et al. ${ }^{11}$ relatam que, entre as pacientes de BLS empregadas à época do tratamento para o câncer de mama, $62,8 \%$ perderam mais de cinco dias no trabalho. Os autores explicam os resultados como sendo decorrentes mais do tratamento sistêmico do que propriamente da cirurgia, já que $41 \%$ delas realizaram QT. Já Burak et al. ${ }^{24}$ verificaram que $70,7 \%$ das 48 mulheres avaliadas relataram retornar às atividades cotidianas ou laborais em apenas três dias após a BLS. No entanto, este estudo foi retrospectivo e pode ter havido viés de memória quando perguntado às pacientes quanto a esta questão, em média, 13 meses após a cirurgia. 
Os estudos de Rietman et al. ${ }^{9,14,15}$, utilizando o Groningen Activity Restriction Scale (GARS), questionário que avalia as incapacidades percebidas em realizar 18 AVDs, mostraram que há restrição na realização das AVDs às seis semanas da cirurgia, mas aos 12 meses a paciente recupera quase que completamente sua capacidade funcional.

Sintomas interferindo na realização de atividades de vida diária foram descritos por Schulze et al. ${ }^{13}$ e relatados por $15,8 \%$ das pacientes estudadas. Também, Barranger et al. ${ }^{17}$ encontraram mais limitações na realização de AVD. Das 54 pacientes estudadas, em média, 24,3 meses da BLS, 27,8\% e 14,8\% delas apontaram restriçōes nas atividades cotidianas ou de trabalho e no lazer, respectivamente. A diferença talvez esteja associada à idade de suas pacientes (58,6 anos, variando de 32 a 80 anos), mais jovens do que no estudo de Schulze et al. ${ }^{13}$. Vinte e sete indivíduos (50\%) eram economicamente ativos antes da cirurgia no estudo de Barranger et al. ${ }^{17}$. Em ambos os estudos, não há relatos quanto ao status laboral da amostra à época da coleta de dados. Leidenius et al. ${ }^{18}$ demonstraram que, após três anos da BLS, 15\% das mulheres narraram que as morbidades de membros superiores, de alguma forma, interferiram em sua rotina diária, sendo que 7\% delas descreveram restrição em atividades domésticas como limpar a casa, lavar pratos ou passar roupas. As mulheres tinham idade entre $31 \mathrm{e}$ 83 anos, com média de 61 anos.

Quando, avaliando quais sintomas interferiram na capacidade de realizar atividades, Thomas-Maclean et al. ${ }^{12}$ encontraram associação entre o desempenho funcional dos membros superiores (medido pelo questionário Disabilites of Arm, Shoulder and Hand - DASH) e a intensidade da dor presente (present pain index do questionário McGill) e entre o DASH e restrição de ADM, especialmente da abdução do ombro. Sua amostra era composta por 343 mulheres, com média de idade de 54,2 $\pm 11,6$ anos tratadas de câncer de mama, avaliadas, em média, oito meses após a cirurgia. Delas, 77 haviam sido submetidas à $\mathrm{BLS}$. Os autores não apresentaram os dados estratificados por tipo de procedimento axilar.

Swenson et al. ${ }^{11}$ perguntaram sobre a interferência das morbidades dos membros superiores (deficiências) nas AVDs, utilizando questionário por eles desenvolvido (Measure of Arm Symptom Survey - MASS) e enviado por correio para 169 mulheres submetidas à BLS com média de idade de 57,9 anos. Após 12 meses da cirurgia, os sintomas relatados como interferindo nas AVDs foram: dor $(9,4 \%)$, dormência (4,3\%), ADM limitada (5,7\%) e edema $(1,4 \%)$. As mulheres mais velhas relataram menos interferência da dor e dormência nas AVDs.
Os sintomas experimentados pelas mulheres submetidas à BLS, em especial a dor, dormência e limitação de ADM, mesmo que de pequena intensidade, interferem de alguma forma na realização das atividades de vida diária, ainda que após três anos da cirurgia. As diretrizes para prática clínica no cuidado e tratamento do linfedema relatam que, embora não haja evidências científicas que sugiram que as atividades repetitivas de grande amplitude de movimento ou que exijam esforço manual promovam ou agravem o linfedema, alguns profissionais de saúde recomendam as pacientes a evitálas, com o intuito de controlar possível sobrecarga do sistema linfático ${ }^{29}$. Portanto, uma pergunta permanece sem reposta: as limitações na realização das AVDs, atividades laborais e de lazer observadas são decorrentes da sintomatologia de membros superiores apresentada ou do receio do desenvolvimento do linfedema?

\section{QUALIDADE DE VIDA (QV)}

A busca, por parte dos profissionais de saúde, de informações sobre qualidade de vida (QV) objetiva nortear estratégias de intervenção terapêutica e criar parâmetros para definição de ações que promovam a saúde individual ou coletiva ${ }^{30,31}$. Não há homogeneidade quanto aos instrumentos e métodos utilizados para avaliar a QV nos estudos. As informações extraídas permitem concluir que a $\mathrm{QV}$ relacionada à saúde em pacientes submetidas à BLS está associada às morbidades físicas apenas no primeiro ano, depois disso o medo da recidiva parece suplantar as limitações funcionais.

Um estudo longitudinal prospectivo não aleatorizado conduzido por Peitinger et al..$^{25}$ analisou as diferenças de morbidade e QV entre 25 mulheres submetidas à BLS e 31 que realizaram LAT. Os resultados mostraram que as escalas Estado de Saúde Global (ESG) e Função Emocional (FE) do questionário European Organisation Research and Treatment of Cancer Quality of Life Questionnaire (EORTC-QLQC30) foram as que apresentaram maior variabilidade antes e após um ano da cirurgia. As mulheres apresentaram melhora da pontuação do Estado de Saúde Global na alta hospitalar após a cirurgia de BLS, o que não aconteceu com a LAT. Transcorridos 12 meses, os escores do ESG, que representam a QV relacionada à saúde, foram de $72,1 \pm 22,74$ numa escala de 0 a 100 , com 100 representando melhor QV. Arnaud et al. ${ }^{20}$ encontraram valores semelhantes (ESG 75,5 $\pm 19,4$ ) ao avaliarem a QV após um ano de cirurgia de BLS em 113 pacientes, comparando-as com LAT. Rietman et al. ${ }^{9}$ relatam que a cirurgia de LAT interferiu significativamente na qualidade de vida, especialmente nas escalas Função Física, Dor, Insônia, Diarreia e Sintomas do Braço. 
Embora seja um estudo comparativo entre LAT e BLS, os autores não apresentam os dados do grupo de BLS.

Barranger et al. ${ }^{17}$, utilizando outro instrumento de medida, em média, 21 meses (10 a 31 meses) da BLS e LAT, não encontraram diferenças estatísticas entre os grupos quanto à QV graduada numa escala de um a dez. Os valores se encontraram entre 7,6 e 7,7 nos grupos, caracterizando boa QV.

Purushotham et al. ${ }^{19}$ realizaram um ensaio clínico aleatorizado, tendo coletado dados antes da cirurgia e em quatro momentos ao longo do período de um ano após a BLS e LAT. Utilizaram questionários validados de avaliação psicológica e de QV, além de medidas da goniometria, perimetria e parestesia de membros superiores. Os resultados mostraram que as pacientes submetidas à BLS apresentaram menores índices de morbidades psicológicas e melhor $\mathrm{QV}$ ao longo de todo o período avaliado e atribuem os melhores escores à menor morbidade física neste grupo, porém não foram apresentadas análises estatísticas que comprovassem sua conclusão.

$\mathrm{O}$ estudo conduzido por Arnaud et al. ${ }^{20}$ identificou que as mulheres mais velhas (média da população $61,1 \pm 11,4$ anos), que não foram submetidas à QT e RT associadas $(79,6 \%)$ e que realizaram mastectomia $(23 \%$ da amostra) apresentavam mais sintomas na mama. $\mathrm{E}$ quanto mais sintomas na mama ou no braço e quanto maior a intensidade da dor, pior era a QV relacionada à saúde após 12 meses da cirurgia. Por outro lado, Fleissig et al. ${ }^{8}$ relataram que as pacientes mais velhas (acima de 65 anos) se recuperaram nos primeiros seis meses da cirurgia mais rapidamente do que as mais jovens. A explicação pode estar associada ao fato de as mulheres mais jovens terem apresentado maior morbidade de membros superiores ou por apresentarem expectativas diferentes. Dubernard et al. ${ }^{21}$ avaliaram a QV de pacientes após, pelo menos, seis meses transcorridos do término da RT, usando o questionário Functional Assessment of Cancer Treatment - Breast Module (FACTB) em 84 mulheres submetidas à BLS. Os resultados mostraram que aquelas abaixo de 55 anos e as que realizaram a LAT apresentaram maior morbidade do braço e consequentemente pior QV. Nenhum fator esteve associado com deterioração do bem-estar social, familiar, emocional e funcional. Makluf et al..$^{30}$, em uma revisão de literatura, afirmam que as mulheres com idade avançada, mesmo quando submetidas à mastectomia, relatam melhor percepção de QV após tratamento do câncer da mama do que as mais jovens. As justificativas estão associadas ao fato de mulheres mais jovens terem maior dificuldade de adaptação após câncer da mama, provavelmente porque as prioridades individuais variam com a idade e os diferentes momentos da vida. Parece que as mais velhas valorizam menos a mama e a feminilidade e têm mais resiliência às adversidades da vida.

No estudo de Leidenius et al. ${ }^{18}$, realizado 36 meses após a BLS, em que foi utilizado um questionário elaborado pelos próprios autores, os sintomas de membros superiores não interferiram na QV das pacientes estudadas. Muitos outros fatores, que não os relacionados à cirurgia, foram considerados angustiantes, como os efeitos da terapia adjuvante (27\%), distúrbios psicológicos como depressão e ansiedade (24\%) e problemas de pele ou fadiga durante a RT (14\%). Apenas 2\% das mulheres que realizaram BLS relatam a morbidade do braço nesse contexto. Baron et al. ${ }^{26}$ também encontraram informações semelhantes. Após cinco anos da cirurgia, seja ela BLS ou LAT, a intensidade da dor ou sua característica não interferiram na QV. Os aspectos descritos como tendo efeito sobre a QV estavam ligados ao medo de recidiva (20\% no grupo BLS e $19 \%$ no LAT) e à imagem corporal (11\% e 17\%). Os autores destacaram que a avaliação da QV foi feita apenas em um momento da pesquisa e é difícil tirar conclusões mais robustas relacionadas a esta questão.

Os aspectos socioculturais podem influenciar os resultados da percepção da QV após a cirurgia do câncer de mama. Não há garantia de que os resultados obtidos em estudos europeus e americanos sejam apropriados para comparação de dados obtidos em pacientes de outras nacionalidades ${ }^{32}$. Pode haver diferenças na percepção da $\mathrm{QV}$, inclusive regionalmente, porque o Brasil é um país marcado por fortes diferenças culturais regionais ${ }^{33}$. Devese ter cautela na comparação de resultados de pesquisas quanto à QV. No Brasil, foi encontrado apenas um estudo que avaliou a morbidade após a BLS, mas não foi estudada a QV entre essas pacientes ${ }^{4}$.

\section{CONCLUSÃO}

Não há instrumento padrão para se avaliar as morbidades após o tratamento do câncer de mama. Isso dificulta a comparação dos resultados e pode explicar a variabilidade na prevalência dos distúrbios apresentados pelas pacientes acompanhadas nos estudos analisados nesta revisão.

Esses estudos indicam evidências de que a morbidade relatada mais frequentemente observada entre pacientes de BLS foi a alteração da sensibilidade da região da axila e mama, que pode perdurar mesmo após cinco anos da cirurgia e é decorrente da transecção do nervo intercostobraquial e seus ramos na região axilar durante o procedimento cirúrgico. 
A dor é o segundo sintoma mais relatado pelas pacientes, embora seja de baixa intensidade.

A ocorrência de linfedema pós-BLS é baixa. Sua prevalência depende do método de avaliação. $\mathrm{O}$ autorrelato da paciente sobre o aumento do volume do membro não deve ser negligenciado, porém é importante diferenciar entre sensação de peso e intumescimento do braço ou perda da sensibilidade. Neste caso, o sintoma estaria relacionado à lesão dos nervos sensitivos axilares.

A BLS pouco interfere na movimentação do ombro. A maior parte das pacientes, segundo as evidências analisadas, retorna às atividades cotidianas, incluindo trabalho e lazer, no primeiro ano da cirurgia. A restrição imposta nesse período parece ser mais em decorrência da QT do que da BLS. Há alguma limitação na realização das atividades cotidianas e de trabalho, mas não se sabe se são os sintomas que causam a restrição ou a paciente que se autolimita com receio de desenvolver o linfedema. Mais estudos são necessários para esclarecer a questão.

A maior recuperação da função do membro superior após a BLS ocorre entre três e seis meses. A exceção são as deficiências na função sensorial, que perduram por um período de até cinco anos. Embora sendo relatado como de ocorrência tardia, não foi observado aumento da incidência de linfedema entre pacientes submetidas à BLS até 36 meses da cirurgia.

A QV relacionada à saúde após a BLS parece ser mais afetada pelos sintomas da mama ou braço nos primeiros 12 meses. Após 36 meses, o medo da recidiva influencia mais os escores de QV do que propriamente as morbidades físicas. Dada a influência dos aspectos socioculturais sobre a percepção que o indivíduo tem de sua saúde, no Brasil faltam estudos que confirmem os resultados encontrados em outros países. Além disso, também são necessários estudos longitudinais para aprofundar conhecimentos sobre os achados dos estudos aqui apresentados.

\section{REFERÊNCIAS}

1. Instituto Nacional de Câncer, Coordenação de Prevenção e Vigilância. Estimativas 2008: incidência de câncer no Brasil. Rio de Janeiro: Instituto Nacional de Câncer; 2007.

2. Langer I, Guller U, Berclaz G, Koechli O, Schaer G, Fehr MK, et al. Morbidity of sentinel lymph node biopsy (SLN) alone versus SLN and completion axillary lymph node dissection after breast cancer surgery - a prospective Swiss multicenter study of 659 patients. Ann Surg. 2007;245(3):452-60.

3. Mansel RE, Fallowfield L, Kissin M, Goyal A, Newcombe RG, Dixon JM, et al. Randomized multicenter trial of sentinel node biopsy versus standard axillary treatment in operable breast cancer: the ALMANAC trial. J Nat Cancer Inst. 2006;98(9):599-609. Erratum in: J Natl Cancer Inst. 2006;98(12):876. Comment in: J Natl Cancer Inst. 2006;98(9):568-9.

4. Magaldi CM, Barros ACSD, Magaldi FM, Mantese JC, Pinotti JA. Avaliação da morbidade e funcionalidade do membro superior em mulheres submetidas à linfadenectomia axilar total e biópsia de linfonodo sentinela por câncer de mama. Rev Bras Mastol. 2005;15(1):9-14.

5. Veronesi U, Paganelli G, Viale G, Path FRC, Luini A, Zurrida S. et al. A randomized comparison of sentinel-node biopsy with routine axillary dissection in breast cancer. $\mathrm{N}$ Engl J Med. 2003;349(6):546-53. Comment in: Lancet Oncol. 2006;7(12):964-6. N Engl J Med. 2003;349(6):603-5. N Engl J Med. 2003;349(20):196871; author reply 1968-71.

6. Veronesi U, Zurrida S. Breast conservation: current status and future perspectives at the European Institute of Oncology. Int J Cancer. 2007;120(7):1381-6.

7. Pater J, Parulekar W. Sentinel lymph node biopsy in early breast cancer: has its time come? [editorial]. J Nat Cancer Inst. 2006;98(9):1023-6. Comment on: J Natl Cancer Inst. 2006;98(9):599-609.

8. Fleissig A, Fallowfield LJ, Langrindge CI, Johnson L, Newcombe G, Dixon JM, et al. Post-operative arm morbidity and quality of life. Results of the ALMANAC randomized trial comparing sentinel node biopsy with standard axillary treatment in the management of patients with early breast cancer. Breast Cancer Res Treat. 2006;95(3):279-93. Epub 2005 Sep 15.

9. Rietman JS, Geertzen JHB, Hoekstra HJ, Dolsma PB, Vries J, Groothoff JW, et al. Long term treatment related upper limb morbidity and quality of life after sentinel lymph node biopsy for stage I or II breast cancer. Eur J Surg Oncol. 2006;32(2):148-52.

10. Armer JMR, Fu JM, Wainstock E, Zagar E, Jacobs LK. Lymphedema following breast cancer treatment, including sentinel lymph node biopsy. Lymphology. 2004;37(2):73-91.

11. Swenson KK, Nissen MJ, Ceronsky C, Swenson L, Lee MW, Tuttle TM. Comparison of side effects between sentinel lymph node and axillary lymph node dissection for breast cancer. Ann Surg Oncol. 2002;9(8):745-53.

12. Thomas-Maclean RL, HackT, Kwan W, Towers A, Miedema B, Tilley A. Arm morbidity and disability after breast cancer: new directions for care. Oncol Nurs Forum. 2008;35(1):65-71.

13. Schulze T, Mucke J, Markwardt J, Schlag PM, Bembenek A. Long-term morbidity of patients with early breast cancer after sentinel lymph node biopsy compared to axillary lymph node dissection. J Surg Oncol. 2006;93(2):109-19.

14. Rietman JS, Dijkstra PU, Geertzen HB, Baas P, Vries J, Dolsma WV, et al. Treatment-related upper limb morbidity 1 year after sentinel lymph node biopsy or axillary lymph 
node dissection for stage I or II breast cancer. Ann Surg Oncol. 2004;11(11):1018-24.

15. Rietman JS, Dijkstra PU, Geertzen JHB, Baas P, Vries J, Dolsma W, et al. Short-term morbidity of the upper limb after sentinel lymph node biopsy or axillary lymph node dissection for stage I or II breast carcinoma. Cancer. 2003;98(4):690-6.

16. Rönka R, Smitten KV, Tasmuth T, Leidenius M. One-year morbidity after sentinel node biopsy and breast surgery. Breast. 2005;14(1):28-36.

17. Barranger E, Dubernard G, Fleurence J, Antoine M, Darai E, Uzan S. Subjective morbidity and quality of life after sentinel node biopsy and axillary lymph node dissection for breast cancer. J Surg Oncol. 2005;92(1):17-22.

18. Leidenius M, Leivonen M, Vironen J, Smitten KV. The consequences of long-time arm morbidity in node-negative breast cancer patients with sentinel node biopsy or axillary clearance. J Surg Oncol. 2005;92(1):23-31.

19. Purushotham AD, Klevesath MB, Bobrow L, Millar K, Myles JP, Duffy SW. Morbidity after sentinel lymph node biopsy in primary breast cancer: results from a randomized controlled trial. J Clin Oncol. 2005;23(19):4312-21.

20. Arnaud S, Houvenaeghel G, Moutardier V, Butarelli M, Martino M, Tallet A, et al. Patients' and Surgeons' perspectives on axillary surgery for breast cancer. Eur J Surg Oncol. 2004;30(7):735-43.

21. Dubernard G, Siderlis 1, Delaloge S, Marsiglia H, Rochard F, Travagli JP, et al. Quality of life after sentinel lymph node biopsy in early breast cancer. Eur J Surg Oncol. 2004;30(7):728-34.

22. Schijven MP, Vingerhoests AJJM, Rutten HJT, Nieuwenhuijzen GAP, Roumen RMH, van Bussel ME, et al. Comparison of morbidity between axillary lymph node dissection and sentinel node biopsy. Eur J Surg Oncol. 2003;29(4):341-50.

23. Temple LKF, Baron R, Cody HS, Fey JV, Thaler HT, Borgen PI, et al. Sensory morbidity after sentinel lymph node biopsy and axillary dissection: a prospective study of 233 women. Ann Surg Oncol. 2002;9(7):654-62.

24. Burak Jr. WE, Hollenbeck ST, Zervos EE, Hock KL, Kemp LC, Young DC. Sentinel lymph node biopsy results in less postoperative morbidity compared with axillary lymph node dissection for breast cancer. Am J Surg. 2002;183:23-7.

25. Peintinger F, Reitsamer R, Stranzl H, Ralph G. Comparison of quality of life and arm complaints after axillary lymph node dissection vs sentinel lymph node biopsy in breast cancer patients. Br J Cancer. 2003;89:648-52.

26. Baron RH, Fey JV, Borgen PI, Stemple MM, Hardick KR, VanZee KJ. Eighteen sensations after breast cancer surgery: a 5-year comparison of sentinel lymph node biopsy and axillary lymph node dissection. Ann Surg Oncol. 2006;14(5):1653-61.

27. Strong, J. Assessment of pain perception in clinical practice. Man Ther. 1999;4(4):216-20.

28. Magee DJ. Avaliação musculoesquelética. 4a ed. São Paulo: Manole; 2005

29. Harris SR, Hugi MR, Olivotto IA, Levine M. Clinical practice guidelines for the care and treatment of breast cancer: 11. Lymphedema. CMAJ 2001;164(2):191-9.

30. Makluf AS, Dias RC, Barra AA. Avaliação da qualidade de vida em mulheres com câncer de mama. Rev Bras Cancerol. 2006;52(1):49-58.

31. Belasco AGS, Sesso RCC. Qualidade de vida: princípios, focos de estudo e intervenções. In: Diniz DP, Schor N. Qualidade de vida: guias de medicina ambulatorial e hospitalar/UNIFESP - Escola Paulista de Medicina. São Paulo: Manole; 2006. p.1-10.

32. Oshumi S, Shimozuma K, Kuroi K, Ono M, Imai H. Quality of life of breast cancer patients and types of surgery for breast cancer: current status and unresolved issues. Breast Cancer. 2007;14(1):63-73.

33. Seidl EMF, Zannon CMLC. Qualidade de vida e saúde: aspectos conceituais e metodológicos. Cad Saúde Pública. 2004;20(2):580-8. 


\section{Abstract}

The second most frequent cancer type worldwide and the most frequent in women is breast cancer. Sentinel lymph node biopsy (SLNB) has become a trend in therapeutic approach nowadays, once it allows disease control with minimal surgical intervention and grants low morbidity rates. This article aims at reviewing literature on musculoskeletal impairments and quality of life among women treated through SLNB for breast cancer. Pubmed, Cochrane, Scielo and Lilacs databases were searched through the years of 1997 to 2008. Medical subject heading terms uses were breast neoplasms, sentinel lymph node biopsy, morbidity and quality of life. Seventy six articles were retrieved and 22 were selected. Results showed the most studied morbidities were sensory disorders $(7 \%$ to $88 \%$ prevalence), pain (3,5\% to $39 \%)$, lymphedema ( $0 \%$ e $22 \%$ ), shoulder range of motion impairment (3\% to $16 \%)$, daily living activities restriction $(7,8 \%$ to $27,8 \%)$ and quality of life deterioration. After 36 months upper limb morbidities hardly seem to interfere with quality of life. Patients have mostly worried about cancer recurrence. Surgery time lapsed and instruments used for data retrieving explain variability in symptoms prevalence. Long term follow-up studies to evaluate morbidities and quality of life research on Brazilian population are missing. Key words: Breast neoplasms; Sentinel lymph node biopsy; Morbidity; Quality of life 